\title{
A method for measuring the presampled MTF of digital radiographic systems using an edge test device ${ }^{\text {a) }}$
}

\author{
Ehsan Sameib) and Michael J. Flynn \\ Department of Diagnostic Radiology, Henry Ford Health System, Detroit, Michigan 48202 and Department \\ of Nuclear Engineering and Radiological Sciences, The University of Michigan, Ann Arbor, \\ Michigan 48109 \\ David A. Reimann \\ Department of Diagnostic Radiology, Henry Ford Health System, Detroit, Michigan 48202
}

(Received 30 December 1996; accepted for publication 13 October 1997)

\begin{abstract}
The modulation transfer function (MTF) of radiographic systems is frequently evaluated by measuring the system's line spread function (LSF) using narrow slits. The slit method requires precise fabrication and alignment of a slit and high radiation exposure. An alternative method for determining the MTF uses a sharp, attenuating edge device. We have constructed an edge device from a $250-\mu \mathrm{m}$-thick lead foil laminated between two thin slabs of acrylic. The device is placed near the detector and aligned with the aid of a laser beam and a holder such that a polished edge is parallel to the x-ray beam. A digital image of the edge is processed to obtain the presampled MTF. The image processing includes automated determination of the edge angle, reprojection, sub-binning, smoothing of the edge spread function (ESF), and spectral estimation. This edge method has been compared to the slit method using measurements on standard and high-resolution imaging plates of a digital storage phosphor (DSP) radiography system. The experimental results for both methods agree with a mean MTF difference of 0.008 . The edge method provides a convenient measurement of the presampled MTF for digital radiographic systems with good response at low frequencies. (C) 1998 American Association of Physicists in Medicine. [S0094-2405(98)00601-4]
\end{abstract}

Key words: modulation transfer function (MTF), resolution, digital radiography, line spread function (LSF), edge spread function (ESF), digital storage phosphor (DSP), computed radiography (CR), image quality, lead, slit, edge

\section{INTRODUCTION}

The resolution properties of an imaging system are commonly described by its modulation transfer function (MTF). ${ }^{1-3}$ The MTF of a radiographic system has been determined by either evaluating the response of the system to periodic patterns, ${ }^{4-6}$ or more commonly, by measuring the line spread function (LSF) of the system using a narrow slit from which the MTF is calculated by Fourier transformation. ${ }^{3,6-16}$ The use of a slit requires very precise fabrication and alignment of the device in the radiation beam, a high radiation exposure to allow sufficient transmission through the narrow slit, and, in most cases, a correction for the finite slit width. ${ }^{14,17}$ Additionally, it is usually necessary to extrapolate the tails of the measured LSF to estimate the low-frequency response. ${ }^{10,14,18}$

An alternative method for determining the MTF of a radiographic system is to measure its edge spread function (ESF) using an opaque object with a straight edge. ${ }^{19-21}$ The ESF is then differentiated to obtain the LSF and the MTF similarly deduced by Fourier transformation. Using a onedimensional theoretical model with no noise reduction, Cunningham and Reid showed that the edge method can be superior to the slit method in measuring the low-frequency response of a radiographic system. ${ }^{22}$ They further showed that the signal-to-noise ratio of the measurement decreases with increasing frequency due to the enhancement of noise by numeric differentiation. In the past, efforts have been made to reduce noise associated with the edge method measurements by convolving the ESF by the Fourier transform of a step function to remove the frequencies above a cut-off spatial frequency; ${ }^{19,23}$ by averaging multiple edge responses and further smoothing of the averaged response by a local rect or Hanning filter; ${ }^{24,25}$ or by fitting the ESF to a parametric equation. ${ }^{17,26-28}$ The former two approaches have not been applied to radiographic systems and the latter approach imposes an a priori mathematical form on the measured MTF and thus prevents observation of any existing detailed structure.

In this article, we report a method for measuring the presampled MTF of digital radiographic systems using a thin, high-precision, attenuating edge test device. The method uses a local smoothing of the ESF to reduce the noise without $a$ priori knowledge of the response function. Signal averaging over a large portion of the edge is further used to obtain MTF results with good signal-to-noise ratio. The method is used to determine the MTF of a digital storage phosphor (DSP) radiographic system and the results are compared to those obtained by a slit method.

One of the complexities in measuring the response of a digital system, as compared to an analog system, is aliasing associated with a discrete sampling. ${ }^{8,29}$ Previous investigators have slightly angulated their slit test devices with respect 
to the pixel array in order to deduce the non-aliased, socalled presampled, ${ }^{8}$ response from various phase shifts of the slit opening with respect to the image array. ${ }^{10,12,14,30-33}$ In order to determine the perpendicular distance from each pixel to the test device, the exact angle and position of the test device must be known. In many previous studies, either the angulation of the test device has been ignored or the method used to determine this angle has not been fully disclosed. In Dobbins et al.,${ }^{14}$ the periodic pattern of the maximum values from a sequence of transverse profiles along the length of a slit was used to determine the slit angle. Most recently, Boone et al. have described an iterative method to deduce an angle of either a slit or a sinusoidal test pattern with respect to the image array. ${ }^{34}$

In this work, the edge device is slightly angulated with respect to the pixel array, as in previous methods using slits. The exact angle of the edge in a digital radiograph of the device is then determined with a precision of $0.02^{\circ}$ using a double Hough transformation followed by an iterative MTF maximization algorithm. To reduce noise, two-dimensional (2D) data from a region along the edge is reprojected to a one-dimensional (1D) array of bins. ${ }^{35}$ Since the 2D data occurs at various distances from the angled edge, the reprojection can be done with bins smaller than the pixel size. Small reprojection bins are used to prevent aliasing in the estimation of the presampled MTF. ${ }^{29}$

\section{EDGE MEASUREMENT METHOD}

In the following subsections, we describe the design and fabrication of a radiographic edge test device, the experimental procedure for aligning the device and acquiring edge images, and the image processing procedure used to obtain the presampled MTF.

\section{A. Edge device design and construction}

An attenuating, thin, high precision edge test device was fabricated to measure the edge spread function (ESF) of the system (Fig. 1). A 250- $\mu$ m-thick lead foil (Goodfellows, Cambridge, England) was used as the attenuating material. The foil was $5 \times 10 \mathrm{~cm}^{2}$ with a purity of $99.95 \%$. For such thickness, the attenuation was assessed to be $85 \%$ for a typical $115 \mathrm{kVp}$ polychromatic x-ray beam using a computational model as described in Sec. III B.

Since lead is soft and can easily be deformed, the lead foil was laminated between two 1-mm-thick slabs of Acrylic using an epoxy-based glue (Git-Rot, Boatlife, Old Bethpage, NY). This particular glue was chosen for its low viscosity which allows a uniform spread of the glue and minimizes the formation of air bubbles in the lamination process. An edge of the laminated lead foil was then milled perpendicular to the flat side of the laminate and polished in multiple steps with decreasing particle size diamond grits down to micronlevel smoothness. Polishing was done with light pressure and at each step the edge was cleaned and examined with a metallurgical microscope for local surface variations and scratches. Special care was taken to prevent embedding the soft surface of the lead with extraneous materials. ${ }^{36}$

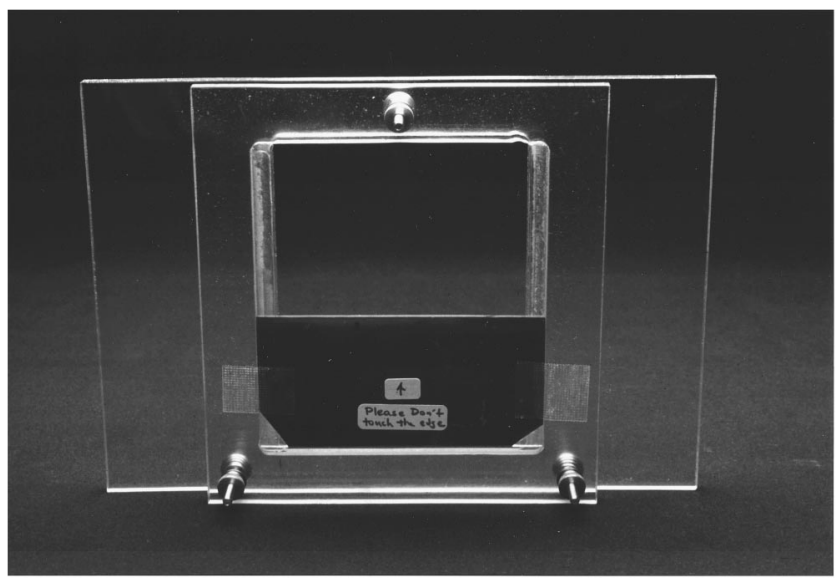

FIG. 1. The edge test device and its holder. The edge device is made of a $250-\mu \mathrm{m}$-thick lead foil laminated between two sheets of Acrylic. The edge of the laminate was milled and polished to form a smooth perpendicular surface. The holder, that was used to position and align the edge device in the x-ray beam, is made of two Lucite frames. The lower, larger frame acts as a base upon which the upper frame and the edge device can be tilted through three adjustment screws.

\section{B. Edge image acquisition}

In order to achieve accurate and reproducible positioning of the edge device in the x-ray beam, a holder was designed and fabricated (Fig. 1). The holder is made of two 6.35-mmthick Lucite frames. The upper frame holds the edge device in place while the lower one acts as a base upon which the upper frame and the edge can be tilted using three adjustment screws.

The alignment procedure is undertaken in three steps, as illustrated in Fig. 2. First, the central axis of the x-ray beam is identified. Small metal markers are placed on the face of
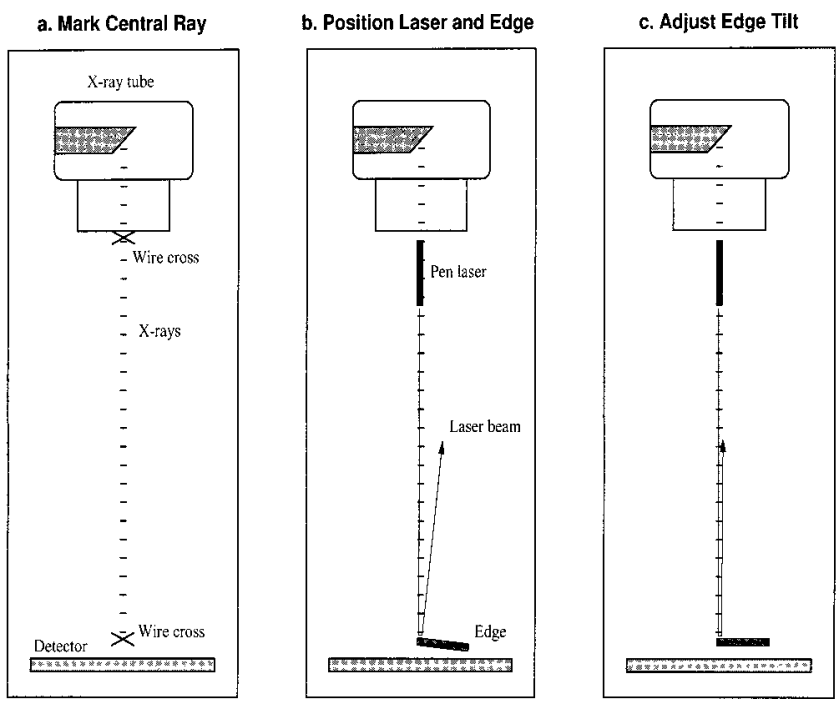

Fig. 2. The schematic of the three-step alignment procedure for the edge device. In the first step, the central axis of the x-ray beam is identified. In the second step, the edge device is positioned at the intersection of the axis with the image plane and a laser beam is pointed toward the edge along the direction of the axis. In the final step, the edge device is tilted until the reflecting laser beam coincides with the incident laser beam. 
the collimator and on the receptor with both positions on the central ray according to the collimator light. A radiograph is taken to identify any misalignment of the central axes of the light beam and the $\mathrm{x}$-ray beam. The edge device is then secured on the holder and placed on the receptor so that the center of its polished edge is at the intersection of the central axis of the x-ray beam with the receptor. The edge is oriented either vertically or horizontally, depending on the direction along which the MTF is being measured, with slight angulation $\left(1^{\circ}-6^{\circ}\right)$.

In the second step, a laser pointer is placed at the face of the collimator with the source of the laser beam on the x-ray central axis. The laser is then pointed to a spot within 1-2 $\mathrm{mm}$ distance from the center of the edge. The reflection of the laser beam off the edge surface is then identified by locating the laser reflection spot on the collimator face. Finally, in the third step, the edge device is tilted with the help of the adjustment screws on the holder until the reflection spot coincides with the incident laser beam. This procedure assures that the polished edge, the source of the laser beam, and the focal spot are all on the same straight line and that the incident $\mathrm{x}$-ray beam is perpendicular to the surface of the receptor and parallel to its polished edge.

After the edge device is properly aligned, a radiograph of the device is taken with an exposure of about 7-9 $\mathrm{mR}$. The radiographic technique factors should allow a transmission of about $5 \%-15 \%$ through the lead (i.e., $90-115 \mathrm{kVp}$ with $3.0 \mathrm{~mm}$ aluminum filtration). A small focal spot, a long source-to-image distance (SID), and the closest possible distance between the edge device and the detector should be used to reduce the focal spot blur.

\section{Edge image processing}

After the image of the edge device is acquired, the digital image data is transferred to a computer and processed to obtain the presampled MTF. As illustrated in Fig. 3, the processing includes one pre-processing and three processing steps, namely angle determination (step 1), reprojection (step 2), and ESF to MTF transformation (step 3), in conjunction with an iterative MTF maximization operation.

In the pre-processing step, an $8 \times 8 \mathrm{~cm}^{2}$ subregion of the acquired image containing only the data from the edge is extracted from the transferred radiograph [Fig. 4(a)]. In a manner similar to a previous work, ${ }^{37}$ a exponential-to-linear transformation is performed on the digital data to obtain the relative deposited-energy data using

$$
I(x, y, E)=k 10^{D(x, y, E) L / 2^{n}},
$$

where $D(x, y, E)$ is the $n$-bit, log-based-10 digital data of the transferred image as a function of its coordinates in the image plane, $x$ and $y$, and the deposited energy in the pixel, $E$; $L$ is the latitude in powers of 10 for the output data; $I(x, y, E)$ is the linear deposited-energy data; and $k$ is a scale factor calculated as $3 \times 10^{4-L}$ to map the data such that the maximum value of $D$ is transformed to a large 16-bit integer

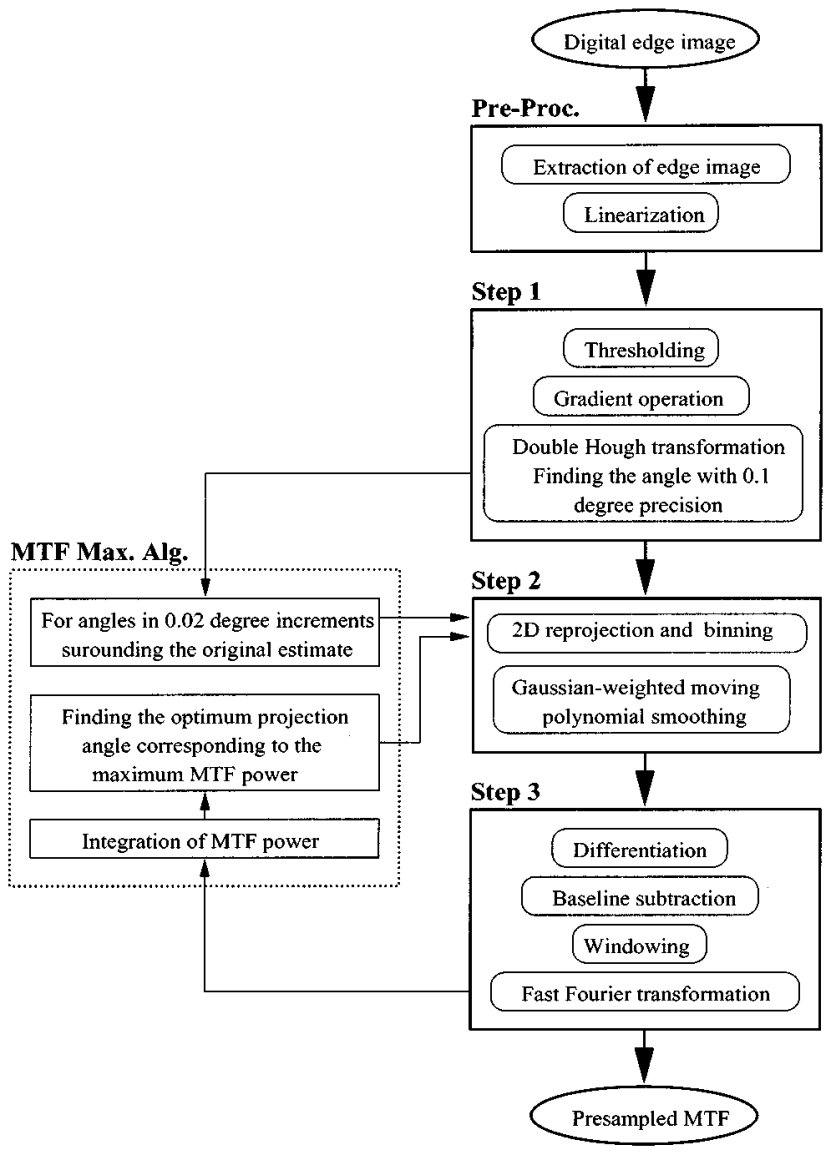

FIG. 3. The processing steps from the digital edge image to the presampled MTF. The data is processed through a pre-processing and three processing steps. The MTF maximization algorithm is added to increase the precision in determination of the edge angle for reprojection of the data to $0.02^{\circ}$.

value. Most digital radiographic systems can provide image data in the form of $D(x, y, E)$; however, the appropriate values for $n$ and $L$ may vary.

In the first processing step, the position and orientation of the edge line in the image are estimated. First, the gray-scale image is converted to an 8-bit binary image by supplying a threshold value computed as the average of the signals from the two sides of the edge image [Fig. 4(b)]. A gradient operation is performed on the 8-bit binary image to acquire a binary line image of the edge transition [Fig. 4(c)]. The angle and position of the edge transition is then found with the aid of the Hough transformation.

The Hough transformation has previously been used as a technique for detection of curves and lines in images. ${ }^{38,39} \mathrm{In}$ this work, the Duda and Hart adaptation of the transform was implemented. ${ }^{40,41}$ Each non-zero data point in the binary image is transformed to a curve in polar coordinate space. The curves associated with collinear data points intersect at a point in the polar coordinate corresponding to the angle and position of the line with respect to a reference point in the image. In practice, the accuracy in determining the angle of a straight line is limited by the discrete sampling in the original image and in the Hough domain. In addition, imperfection in the straightness of the line may cause the intersection 


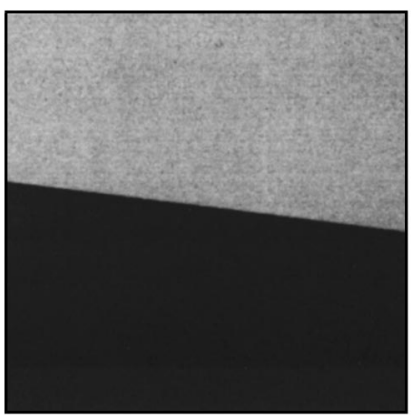

(a)

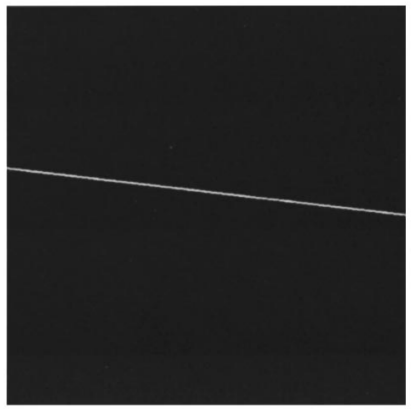

(c)

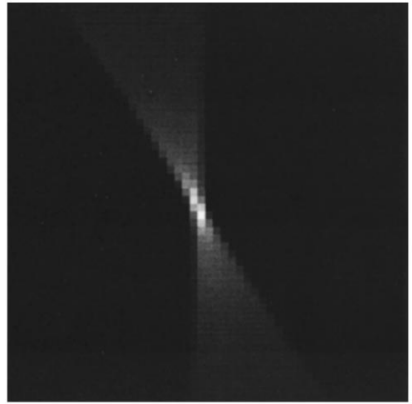

(d)

FIG. 4. Edge angle determination. (a) An edge image block extracted from a radiograph of the edge test device (techniques are as specified in Fig. 11). (b) The output of the thresholding operation of the edge image. c) The output of the gradient operation. The one-pixel-thick white line in the image is the segmented edge transition. (c) The results of the second Hough transformation of the binary line image. The maximum of the converging pattern in the center of the image identifies the position and angle of the edge transition in the polar coordinate with an angular precision of $0.1^{\circ}$.

point to "spread.' In such cases, the best estimates for the angle and the position of a straight line can be found by identifying the maximum of the intersecting pattern in the Hough domain [Fig. 4(d)].

In order to assure sufficient precision in the estimation of the angle and position of the edge transition, the gradient line image is passed through the Hough transformation twice. First, the binary line image is transformed within a $\pm 180^{\circ}$ range with an angular sampling of $1^{\circ}$. A second pass through the transformation is then carried out within a $\pm 10^{\circ}$ subregion surrounding the first estimate to obtain the edge angle with an improved precision of $0.1^{\circ}$ [Fig. 4(d)].

In the second processing step, the ESF is obtained by reprojecting the gray-level data of the original linearized image along the direction of the estimated angle into a onedimensional array of sub-pixel elements. ${ }^{35}$ Since the edge is placed obliquely with respect to the pixel array, individual rows across the edge are shifted relative to the edge position. The average of a large number of rows with a phase shift can be used to acquire the non-aliased (or presampled) ESF of the system, as explained below.

Referring to Fig. 5, if individual rows are reprojected at the correct angle of the edge, for each row reprojected, the true ESF of the system as a function of the distance from the edge, $s$, is sampled as
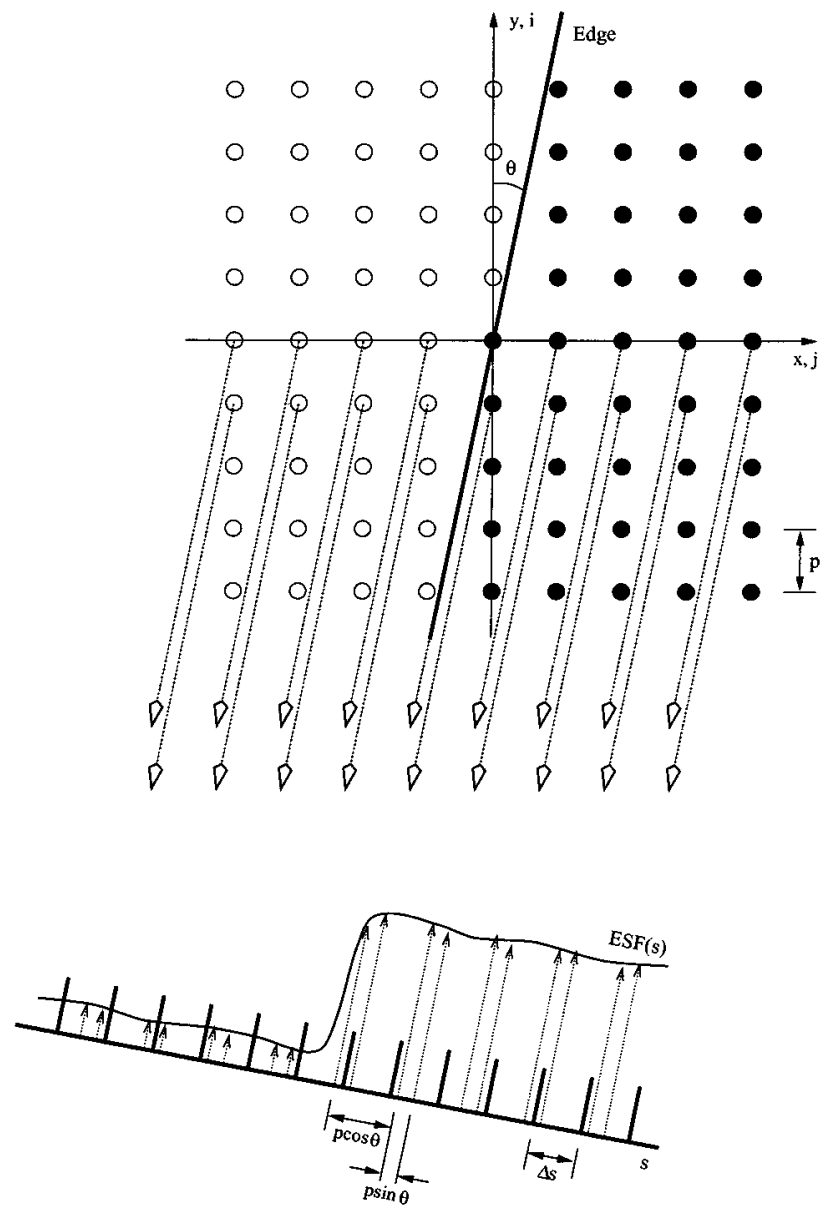

FIG. 5. Reprojection and binning of the two-dimensional edge image data into a one-dimensional trace perpendicular to the edge.

$$
E_{j}^{i}=\int \operatorname{ESF}(s) \delta(s+i p \sin \theta-j p \cos \theta) d s,
$$

where $E_{j}^{i}$ is a set of discrete samples of the function $\operatorname{ESF}(s)$ for pixels in the $i$ th row at a distance $s(i, j)=p(j \cos \theta$ $-i \sin \theta$ ) from the edge, $i$ is the row number, $j$ is the column number, $p$ is the pixel dimension which is assumed to be the same in both orthogonal directions, and $\theta$ is the reprojection angle. $E_{j}^{i}$ array corresponding to different rows, $i$, are displaced by varying amounts in the distance $s(i, j)$, as illustrated in Fig. 5. When $N$ rows are reprojected, the sampling points are accumulated on the $s$ axis, forming a composite pattern of finely spaced, discrete samples of the ESF, $E_{i j}$. In general, these samples are not uniformly distributed over the $s$ axis. The spacing distribution depends on the pixel dimension, $p$, the size of the image array, $N$, and the reprojection angle, $\theta$. The $E_{i j}$ samples can be reordered and collected into bins with a spatial width of $\Delta s$ as

$$
\mathrm{ESF}_{k}=\frac{1}{n_{k}} \sum_{i j} E_{i j} \operatorname{bin}(s(i, j)-k \Delta s),
$$

where $n_{k}$ is the number of pixels whose distance from the edge, $s(i, j)$, falls within $\left(k-\frac{1}{2}\right) \Delta s$ and $\left(k+\frac{1}{2}\right) \Delta s$, and 
$\operatorname{bin}(s(i, j)-k \Delta s)$ is a rectangle function defined to have a value of 1 for $|s(i, j)-k \Delta s| \leqslant \Delta s / 2$, and zero, elsewhere.

In general, for a finite image array and a small reprojection angle, $n_{k}$ varies for different bins. For a given reprojection angle, the variation in $n_{k}$ depends on the bin width, $\Delta s$, and the pixel dimension, $p$. If $\Delta s \sim p$, a large number of reprojected pixels are averaged within each bin and the reprojection/binning operation is equivalent to a periodic sampling of the presampled ESF with a large, uniform aperture. Noise in the measured ESF is small due to averaging of a large number of pixels, but the large aperture causes poor spatial frequency response. If $\Delta s \ll p$, the average number of reprojected pixels within each bin is markedly reduced; the ESF will be finely sampled, but noise may become objectionable. For extremely small $\Delta s$, some bins may accumulate no pixels. To avoid these two extreme cases, the $\Delta s / p$ value can be devised to accumulate a sufficiently large number of pixels within each bin while still providing good frequency response.

In this work, a sub-pixel spatial bin width of $\Delta s=0.1 p$ was used, which was ascertained to provide an acceptable trade-off between the sampling uniformity and noise, on one hand, and sampling frequency, on another hand. This subpixel sampling rate was also found to be sufficient to prevent errors associated with finite-element differentiation ${ }^{21}$ and undersampling. ${ }^{29}$ The reprojection is done along a $5 \mathrm{~cm}$ length of the edge in a $5 \times 5 \mathrm{~cm}^{2}$ block surrounding the midpoint of the edge line. For image arrays with 200 and 100 $\mu \mathrm{m}$ pixel dimensions, this results in approximately 25 and 50 pixels to be reprojected and averaged within each bin, respectively. These numbers remain reasonably constant as the reprojection angle varies. Consequently, in our method, as opposed to more angle-sensitive methods, ${ }^{33}$ there are no specific requirements for the relative angle of the edge with the image array; the edge can be placed at any angle. The only exceptions are angles $\theta=\tan ^{-1}(1 / n) \pm k(\pi / 2)$ for which one pixel in one direction (horizontal or vertical) corresponds to exactly $n$ pixels in its perpendicular direction, where $n$ is an integer between 0 and 9 for a subpixel bin width of $\Delta s$ $=0.1 p$. Zero and 45 degrees are such exceptions.

The $\mathrm{ESF}_{k}$ array is then smoothed by utilizing a fourthorder, Gaussian-weighted, moving polynomial fit [Fig. 6(a)]. The use of local smoothing does not confine the ESF to a particular mathematical form. For each element in the $\mathrm{ESF}_{k}$ array, a polynomial function is fit using adjacent elements and the initial element value is replaced with the value predicted by the fit. A least-squares fit is employed for which values near the center-point are strongly weighted by entering a different variance value for each point. ${ }^{42}$ The weighting function is a Gaussian in the form of

$$
f(i)=\exp \left[-\left(\frac{4 i}{w-1}\right)^{2}\right]
$$

where $w$ is the window width and $i$ is a local variable defined within $[-(w-1) /(2),(w-1) /(2)]$. A fixed window width of 17 elements was used in this study. The polynomial order

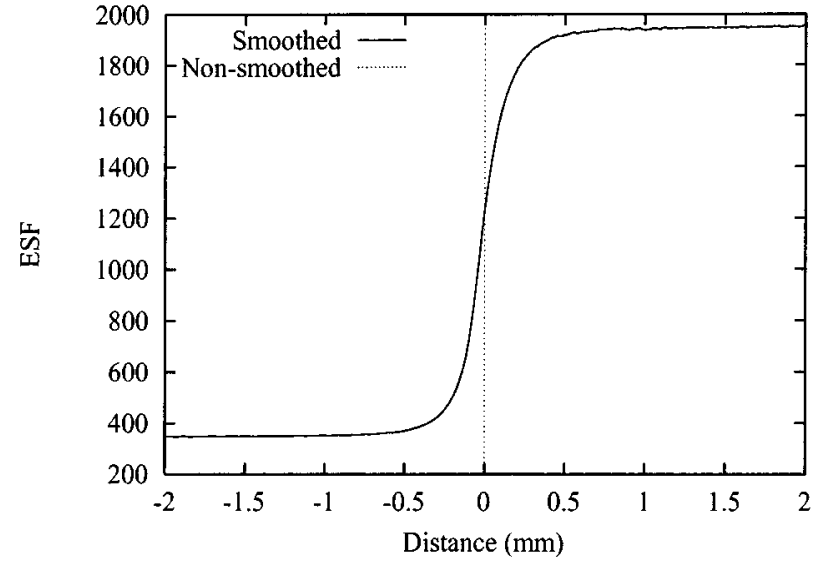

(a)

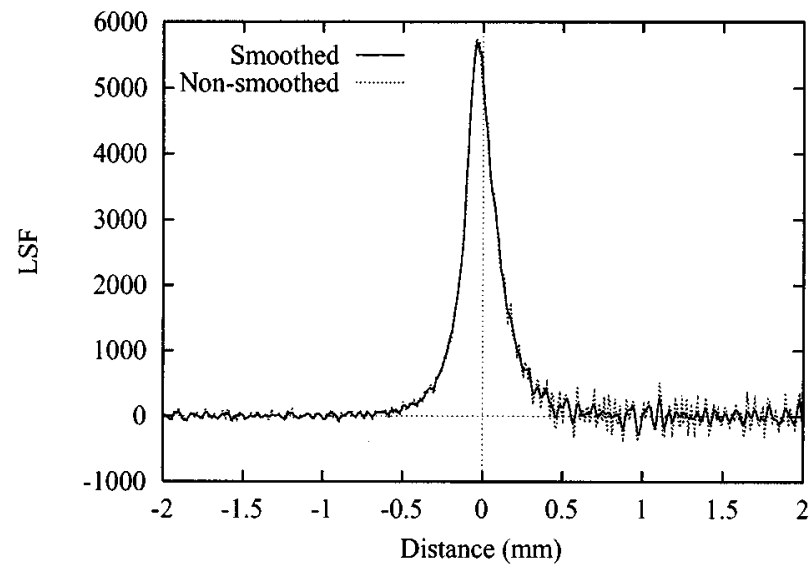

(b)

FIG. 6. (a) The ESF computed from Fig. 4(a) obtained by reprojecting the linearized data along the edge direction into a perpendicular trace and resampling it into 0.1 subpixel bins. The smoothed ESF was obtained by a Gaussian-weighted moving 4th-order polynomial fit with a window width of 17 bins (1.7 pixels). The slight effects of the smoothing can be observed at the "attenuated" side of the ESF (note the reverse relationship between the data and the exposure level). (b) The LSF obtained by numerical differentiation of the smoothed and non-smoothed ESFs. The effects of the ESF smoothing are much more apparent in the LSF.

and the window width were chosen based on a test performed on simulated edge images discussed in Sec. III B.

In the third processing step, the smoothed $\mathrm{ESF}_{k}$ array is processed to obtain the presampled MTF. First, the array is numerically differentiated with a standard central-difference algorithm to obtain the LSF [Fig. 6(b)] using

$$
\mathrm{LSF}_{k}=\frac{\mathrm{ESF}_{k+1}-\mathrm{ESF}_{k-1}}{2 \Delta s} .
$$

To remove the effects of low-frequency nonuniformities often associated with the heel effect, the baseline of the LSF is subtracted using a linear fit to the 10 -nm-long portions of the LSF tails. The baseline subtraction is different from the extrapolation of the LSF tails in several respects. First, the data used for baseline correction is outside the range of the data used to determine the MTF from the LSF; the complete LSF data has a spatial extend of about $50 \mathrm{~mm}$ from which only 
the central $20 \mathrm{~mm}$ region is used to compute the MTF (consider the Hanning window width described below). Second, this process only removes the background trend, affecting only the lowest frequencies in the MTF, without imposing any functional form to the tails.

After correcting for the baseline trend, a Hanning filter ${ }^{41}$ with a window width of $20 \mathrm{~mm}$ is applied to the LSF to establish a sampling rate of 0.05 cycles $/ \mathrm{mm}$ in the frequency domain and to eliminate the high-frequency content of the measurement not associated with the edge transition (i.e., noise in the tails of the LSF). The presampled MTF is then obtained by a fast Fourier transform (FFT) $)^{41}$ of the LSF. The MTF is normalized to its value at zero frequency.

The presampled MTF obtained through the three processing steps described above is very sensitive to the determination of the edge angle. The precision in the edge angle determination is further improved to $0.02^{\circ}$ by an iterative MTF maximization algorithm (Fig. 3). 17 different MTFs are computed for different reprojection angles within a $\pm 0.16^{\circ}$ subregion surrounding the estimate obtained by the double Hough transformation. Each MTF is integrated within the spatial frequency interval of $0-2$ cycles $/ \mathrm{mm}$. The angle associated with the maximum of the MTF integral is then identified as the best estimate for the edge angle, and its corresponding MTF, for the final result. It should be noted that for most cases, the result of the MTF maximization algorithm is identical to or within $\pm 0.04^{\circ}$ of that obtained by the double Hough transformation.

\section{EVALUATION OF THE METHOD USING SIMULATED IMAGES}

We have used computer-simulated images of an edge device to evaluate different processing steps of our method. Within $512 \times 512$ pixel array, the edge transition was defined by a $5.5^{\circ}$ straight line passing through the center of the image dividing it into two distinct regions with different average pixel values. The simulated images were formed both with constant pixel values at these regions and with superimposed Poisson statistical fluctuations surrounding the average pixel value in each region.

\section{A. Simulated edge image without noise}

An edge image with constant pixel values of 100 and 1000 corresponding to an edge device with $10 \%$ transmission was analyzed using the methods described in Sec. II C. A pixel size of $200 \mu \mathrm{m}$ was assumed and no smoothing was used for the ESF. Figure 7 illustrates the resultant ESF, LSF, and presampled MTF. The ESF is very similar to a step function, differing only in that an intermediate value appears at the edge transition due to the discrete nature of the analysis method. The numerical differentiation of the ESF leads to a LSF with a triangular shape and a width of four subpixels $(0.08 \mathrm{~mm})$, as illustrated in Fig. 7(a). The Fourier transform of a triangle function with a total width of $4 \Delta s$ is a sinc ${ }^{2}$ function with its first zero value at $1 / 2 \Delta s .{ }^{43}$ Similarly, the MTF evaluated from this simulated image is very similar to a sinc ${ }^{2}$ function with it first zero at 25 cycles/mm [Fig. 7(b)].

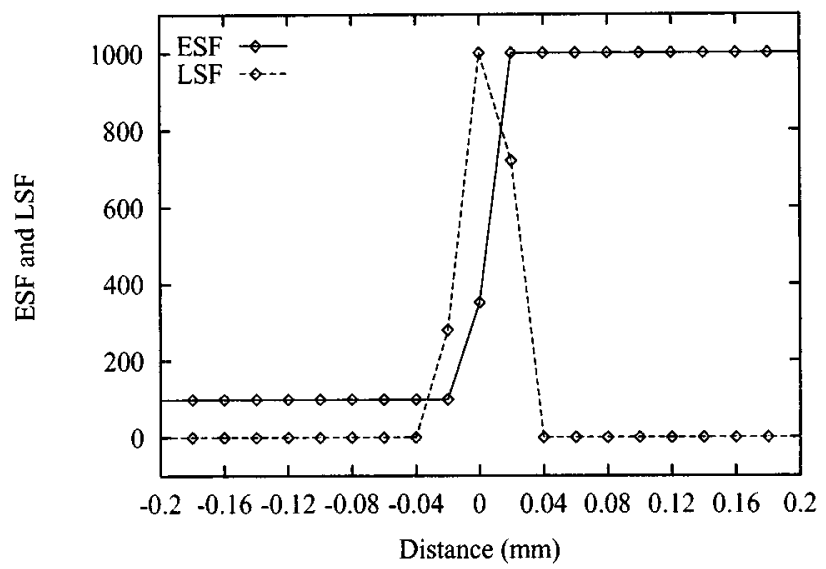

(a)

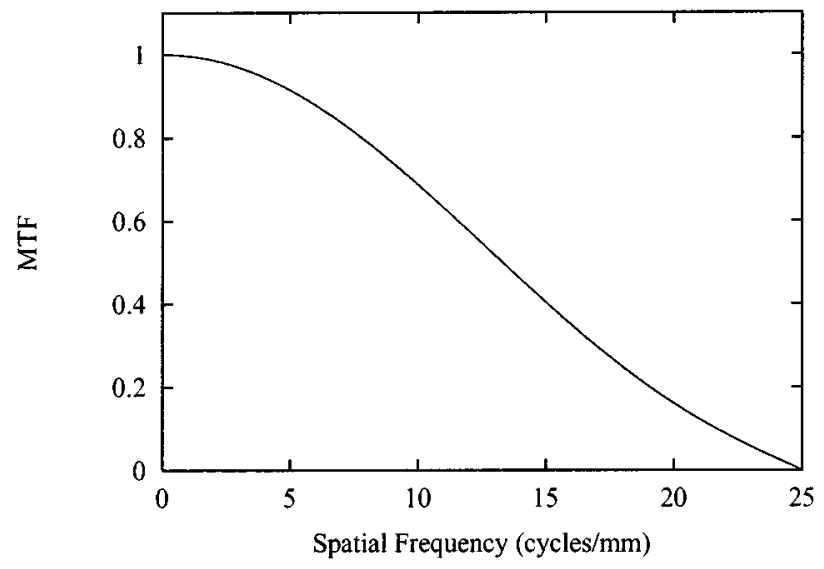

(b)

FIG. 7. The ESF, LSF (a), and presampled MTF (b) obtained by processing a simulated edge image with a step-function edge transition and constant pixel values.

In this idealized case, the dampening of high frequencies is less than $9 \%$ within $0-5$ cycles $/ \mathrm{mm}$, the frequency range that is relevant to most current radiographic applications. A finer subbinning of the reprojected ESF data would reduce the high-frequency dampening effect at the expense of a higher level of noise as discussed in Sec. II C.

The simulated noise-less image was also used to observe the error in the computed MTF as a result of error in the reprojection angle. In a sequence of runs, the reprojection angle was artificially altered in $0.01^{\circ}$ increments around the angle correctly estimated by the angle determination algorithm $\left(5.5^{\circ}\right)$. No smoothing was used for the ESF. Figure 8 illustrates the results. For errors in the reprojection angle more than $\pm 0.04^{\circ}$, multiple intermediary values appear in the ESF transition and the zero of the MTF occurs at a lower frequency. Based on these results, a precision of $0.02^{\circ}$ was considered sufficient for the angle determination. As described in Sec. II C, this precision was achieved by an iterative MTF maximization algorithm following a double Hough transformation. 


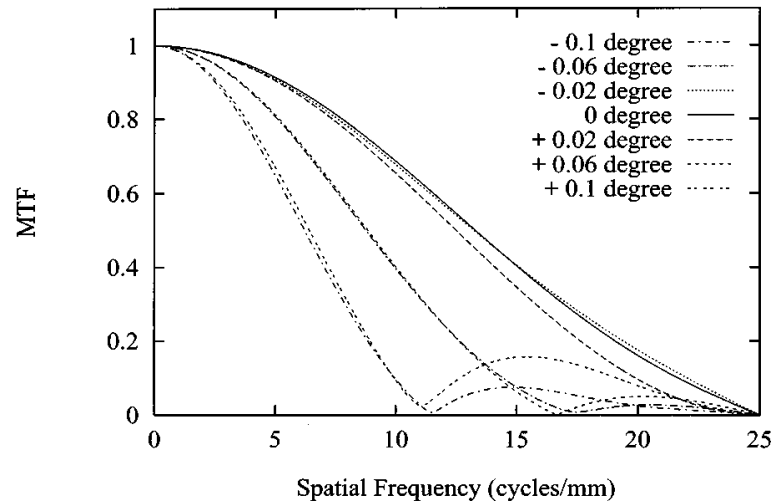

FIG. 8. The presampled MTF of a noise-less simulated edge image as a function of deviation of the reprojection angle from the true angle of the edge in the image.

\section{B. Simulated edge image with noise}

The influence of quantum noise in the image is of concern for any method of measuring the MTF for radiographic systems. Computer-simulated images of an edge device into which Poisson noise was added were used to evaluate the propagation of quantum image noise to the MTF in our method. Simulated images were first made as in Sec. III A

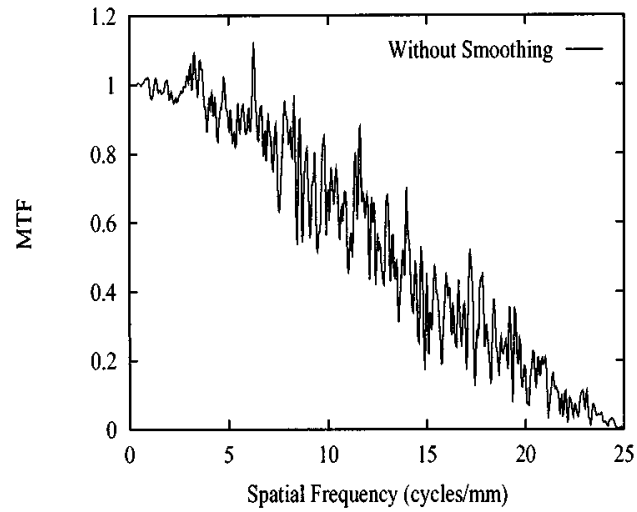

(a)

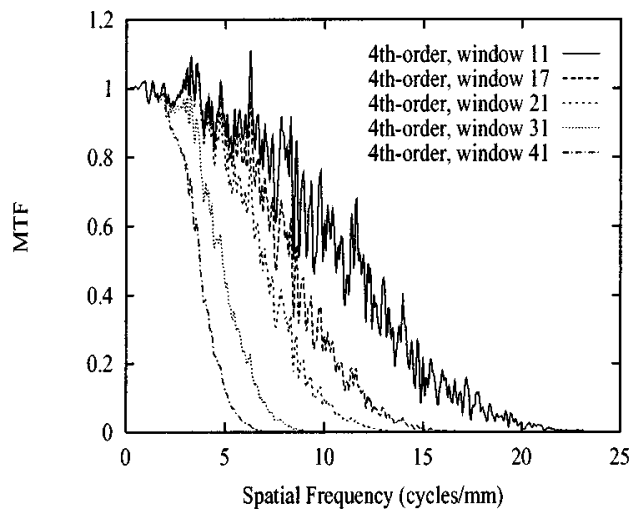

(c) with values of 1000 for all the pixels in the nonattenuated region of the image and $1000 t$ for all the pixels in the opaque region ( $t=$ transmission). Random fluctuations were then added to each pixel by sampling a Poisson distribution function having a mean equal to the pixel value in the region (i.e., 1000 or $1000 t$ ) using a random number generator. For 200 $\mu \mathrm{m}$ pixels, this corresponds to a noise equivalent quanta (NEQ) of $25000 \mathrm{~mm}^{-2}$, about 1/10th the value encountered experimentally in radiographic applications.

In order to choose the polynomial order and the window width for our Gaussian-weighted, moving polynomial smoothing of the ESF, a simulated edge image with noise having a $10 \%$ transmission $(t=0.1)$ was analyzed with various combinations of these smoothing parameters. Only evenorder polynomials were examined. Figure 9 shows the resultant MTFs. It is evident that more rigorous smoothing of the ESF produces a less noisy MTF at the expense of dampening the high-frequency response. From these results, a fourthorder polynomial and a window width of 17 subpixels (1.7 pixels) were identified to reduce noise while maintaining the dampening of the high-frequency response within the $0-5$ cycles/mm frequency range to less than 13\%. The FWHM of this window width is approximately 6.7 subpixels (0.67 pixels).

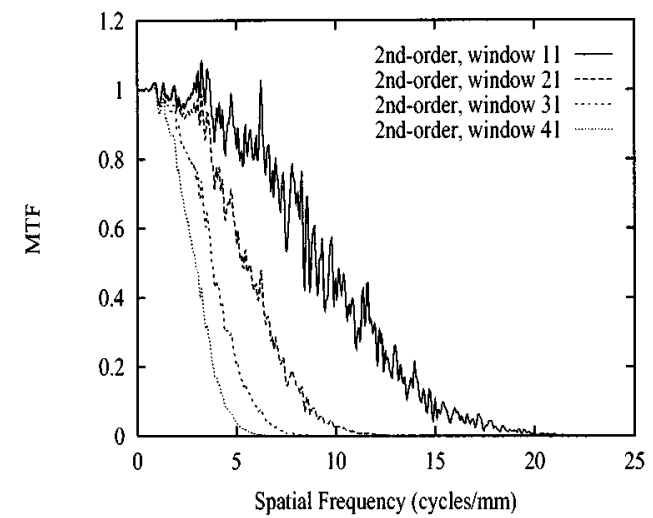

(b)

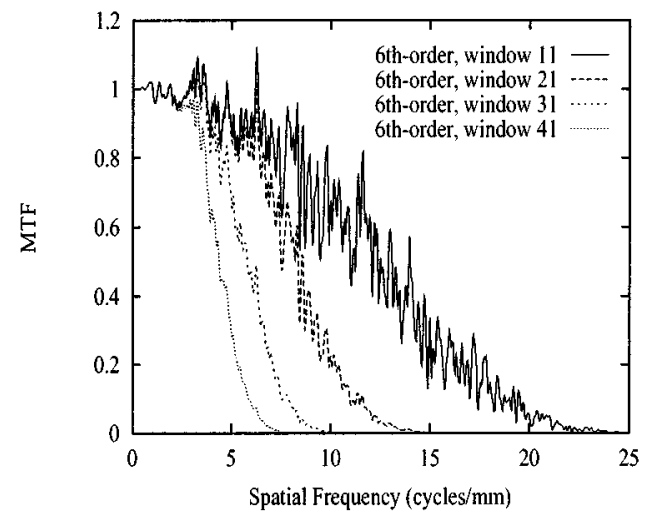

(d)

FIG. 9. The effects of the smoothing parameters for the Gaussian-weighted moving polynomial fit of the ESF on the resulting presampled MTF. The data were obtained from a simulated edge image with Poisson noise superimposed, corresponding to an edge transmission of $10 \%$ and a noise equivalent quanta (NEQ of $25000 \mathrm{~mm}^{-2}$. (a) Without smoothing, (b) 2nd-order polynomial, (c) 4th-order polynomial, and (d) 6th-order polynomial. The window widths are specified in units of subpixels ( 1 subpixel $=0.1$ pixel). 


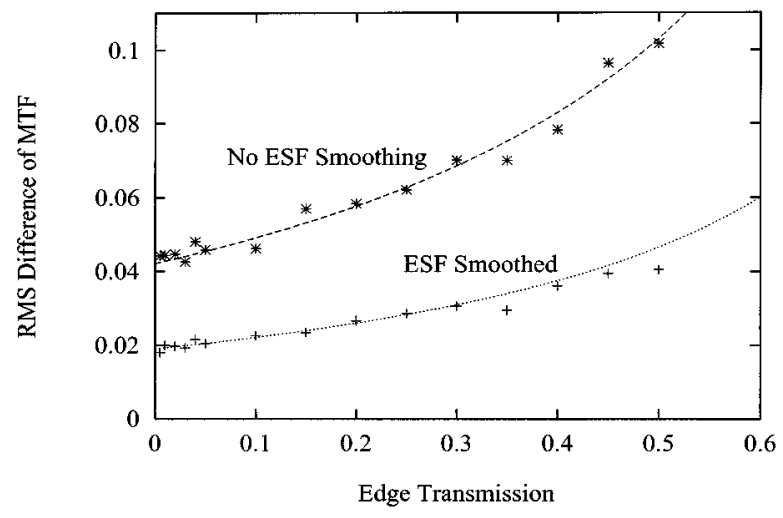

FIG. 10. The root-mean-square (rms) difference of the presampled MTF, calculated with respect to the ideal case of Fig. 7(b), as a function of edge transmission. The data were obtained using simulated edge images with Poisson noise superimposed, corresponding to various edge transmission values and a noise equivalent quanta (NEQ) of $25000 \mathrm{~mm}^{-2}$.

A consideration when designing an edge device for testing radiographic systems is the edge transmission, dictated by its thickness and the applied beam quality. In order to determine the influence of edge transmission in our method, simulated edge images with Poisson noise superimposed were generated corresponding to edge devices with different transmissions. The images were analyzed and the root-meansquare (rms) of the difference between each presampled MTF and the ideal response of Fig. 7(b) was calculated. Results were obtained both without smoothing of the ESF and with smoothing, using the smoothing parameters specified above. Figure 10 shows the calculated rms difference as a function of edge transmission. The rms noise measure is expected to be directly proportional to average noise and inversely to the edge contrast. The data of Fig. 10 was thus fit to an equation of the form

$$
\operatorname{rms}(t)=\frac{c}{\sqrt{N_{0}}}\left(\frac{\sqrt{1+t}}{1-t}\right),
$$

where $t$ is the edge transmission, $N_{0}$ is the average pixel value in the non-attenuated side of the edge image, and $c$ is a proportionality constant. The values for $c$ in the measured MTF with and without ESF smoothing were determined to be 0.6 and 1.33 , respectively.

In general, the noise in the MTF increases with the edge transmission. As shown in Fig. 10, high transmission values can lead to objectionable noise in the measured MTF as a result of signal reduction. However, transmission values of less than a few percent are experimentally impractical. In practice, the edge transmission should be less than 0.5 , and a transmission less than 0.2 will insure that the noise is within $30 \%$ of its minimum value at $t=0.0$. In this work, we used a 250 - $\mu \mathrm{m}$-thick lead edge device. Using a semiempirical x-ray spectra model that accounts for both characteristic x-ray and bremsstrahlung radiation, ${ }^{44,45}$ the transmission through 250 $\mu \mathrm{m}$ lead was predicted to be within 0.05 to 0.15 for a $90-$ $115 \mathrm{kVp}$ x-ray beam.

\section{EXPERIMENTAL COMPARISON OF EDGE AND SLIT METHODS}

\section{A. Comparison methods}

Ideally, the experimentally determined presampled MTF of a radiographic system should be the same whether measured with an edge device or a slit device. To examine this, our edge method and an established slit method were applied to the same radiographic system. The measurements were performed at the Health Imaging Research Laboratory of the Eastman Kodak Company using a digital storage phosphor (DSP) radiography system (Kodak Ektascan Storage Phosphor Reader, Model-400). A $18 \times 24 \mathrm{~cm}^{2}$ standard resolution, General Purpose, imaging plate (Kodak, GP-25) and a similar size high resolution, High Definition, imaging plate (Kodak, HD) were employed for both measurements using a pixel size of $100 \mu \mathrm{m}$. The presampled MTF was determined in both the laser-scan and the plate-scan directions.

The edge images were acquired using the acquisition procedure specified in Sec. II B using a $90 \mathrm{kVp}$ x-ray beam with $3.0 \mathrm{~mm}$ added aluminum filtration, $5.0 \mathrm{mAs}$, a $0.6 \mathrm{~mm}$ focal spot size, and a source-to-image distance (SID) of $183 \mathrm{~cm}$. The exposure was measured in air to be $9.5 \mathrm{mR}$ at the edge surface. The $1792 \times 2392$ array of 16-bit-stored, 12-bit logscaled data were transferred to a Unix workstation (Sun Sparc 2) and linearized using Eq. (1) with a latitude $(L)$ value of 4.0. The edge images were then processed as outlined in Sec. II C to obtain the presampled MTF of the system for each specified direction.

The slit measurements were performed on identical imaging plates using the angulated slit technique described by Fujita et al. ${ }^{33}$ A dedicated, prealigned slit exposure apparatus originally designed for precise laboratory measurement of the MTF for conventional screen-film systems was used to acquire the slit images. ${ }^{3}$ This slit aperture was made of two 2-mm-thick, 12.5-mm-long platinum jaws. The slit width was $10 \mu \mathrm{m}$. Since in this experimental setup the slit device was fixed, a cassette angulation device was added behind the slit to rotate the storage phosphor cassette about $1.5^{\circ}$ in order to create an apparent angulated slit while preserving the integrity and alignment of the slit exposure device. The exposures for the measurements in the laser-scan and the platescan directions were acquired by $90^{\circ}$ rotation of the cassette. The slit exposures were made using a $90 \mathrm{kVp}$ x-ray beam with $3.0 \mathrm{~mm}$ added aluminum filtration (identical to the edge measurements), $160 \mathrm{mAs}$, a $1.2 \mathrm{~mm}$ focal spot size, and a source-to-image distance (SID) of $72 \mathrm{~cm}$. The mAs was chosen so that the tails of the LSF were represented down to approximately $0.2 \%$ of the LSF maximum.

From the image data, a composite LSF was synthesized using a method similar to Fujita et al. ${ }^{33}$ First the data in the vicinity of the slit center $( \pm 2.5 \mathrm{~mm})$ was converted to a linear scale using a log latitude of 4 . Each individual scanline LSF was fit to a functional form using a nonlinear leastsquares algorithm. ${ }^{42}$ The fitting function was a convolution of an exponential and a Gaussian function (note that this form is different from a combination of these functions as used by Yin et $a l .{ }^{32}$ ). The exponential function represents the 


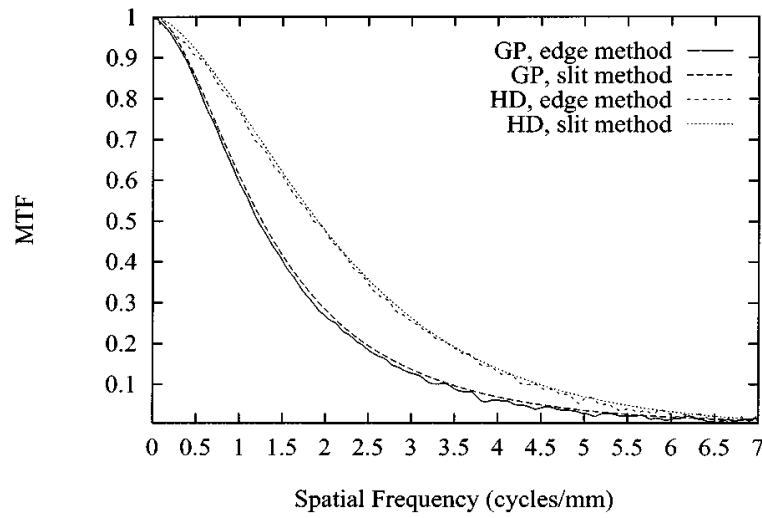

FIG. 11. The presampled MTF of a digital storage phosphor system (Kodak Ektascan Storage Phosphor Reader, Model-400) for two kinds of phosphor plates (Kodak, GP-25 and HD) determined using the edge and slit methods in the laser-scan direction. Identical phosphor plates, readout pixel size (100 $\mu \mathrm{m})$, and beam quality ( $90 \mathrm{kVp}, 3.0 \mathrm{~mm}$ aluminum filtration) were used in all the measurements. The results from the slit method are averages of three independent measurements, while those from the edge method are from single measurements. (Edge method: $0.6 \mathrm{~mm}$ focal spot, SID $183 \mathrm{~cm}, 5.0$ mAs; Slit method: $1.2 \mathrm{~mm}$ focal spot, SID $72 \mathrm{~cm}, 160 \mathrm{mAs}$ ).

scattering of the laser beam within the phosphor while the Gaussian function represents either the Gaussian profile of the laser beam in the plate-scan direction and/or the higher order Bessel filter used as an antialiasing filter in the laserscan direction. Consequently, the convolution of these functions provides a very good representation of the line spread function for storage phosphor systems. This fit was used to predict the peak location of each undersampled LSF as well as the peak amplitude, baseline offset, and the characteristic widths of the exponential and the Gaussian functions. A linear least-squares fit to the peak location for each scan line in the slit was then used to calculate the angle of the slit. The composite LSF was subsequently calculated by reprojecting the individual undersampled LSFs along the direction of the estimated angle into a one-dimensional array. A discrete Fourier transformation (DFT) was then performed on the baseline-corrected LSF data, normalized at zero spatial frequency, to obtain the presampled MTF. It should be noted that since the error in the MTF due to the finite slit width was less than $1 \%$ up to the spatial frequency of 5 cycles $/ \mathrm{mm}$ (assuming a rect function for the slit aperture), no sinc correction was applied in these measurements.

\section{B. Comparison results}

Figures 11 and 12 show the measured presampled MTF in the laser-scan and the plate-scan directions for the GP and HD plates using the edge and slit methods. The directions are approximate since the devices were slightly angulated from the true horizontal and vertical directions. The slit method results are averages of three independent measurements on the same imaging plates, while those from the edge method are from single measurements. For both the GP and the HD plates, the MTFs in the plate-scan direction were slightly higher than those in the laser-scan direction, similar to previous measurements on DSP systems. ${ }^{14}$ In both directions,

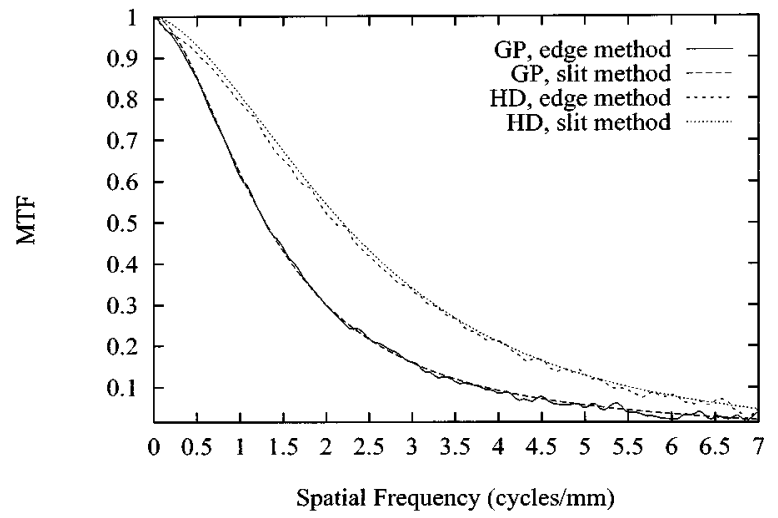

FIG. 12. The presampled MTF of a digital storage phosphor system (Kodak Ektascan Storage Phosphor Reader, Model-400) for two kinds of phosphor plates (Kodak, GP-25 and HD) determined using the edge and slit methods in the plate-scan direction (also see the caption of Fig. 11).

the HD plate exhibited significantly higher modulation response than the GP plate since a HD plate is approximately half as thick as a standard GP plate. ${ }^{46}$

The results for the edge and slit methods were similar. Within the frequency range of $0-5$ cycles $/ \mathrm{mm}$, the MTF values obtained with the two methods were compared at 0.01 cycles $/ \mathrm{mm}$ intervals. For a given spatial frequency, the MTF response from the slit method was slightly higher than that from the edge method. The MTF differences observed for all measurements (i.e., the GP and HD plates in both directions) varied within $[-0.008,0.025]$ with a mean difference of 0.0085 and a root-mean-square (rms) difference of 0.01093 .

For a given MTF response, the spatial frequency from the slit method was slightly higher than that from the edge method. Table I tabulates the measured frequencies at MTF values of 0.5 and 0.1 . On a relative scale, the edge method results had less than $2 \%$ deviation from the slit method results, with a range extending from approximately $1.5 \%$ at lower frequencies to about $3 \%-4 \%$ beyond the Nyquist frequency.

\section{DISCUSSION}

In order to objectively evaluate and compare the performance of digital radiographic systems and to provide essential information for image enhancement, accurate and convenient techniques are required to assess the MTF of these

TABLE I. A comparison of MTF measurement for a digital radiographic system (Kodak Ektascan Storage Phosphor Reader, Model-400) acquired by the slit and the edge methods in the laser-scan and the plate-scan directions (also see the caption of Fig. 11).

\begin{tabular}{llllll}
\hline \hline & \multicolumn{2}{c}{ GP Plate } & & \multicolumn{2}{c}{ HR Plate } \\
\cline { 2 - 3 } \cline { 5 - 6 } & $\begin{array}{llllll}\text { Laser-scan } \\
\text { cycles/mm }\end{array}$ & $\begin{array}{l}\text { Plate-scan } \\
\text { cycles/mm }\end{array}$ & & $\begin{array}{l}\text { Laser-scan } \\
\text { cycles } / \mathrm{mm}\end{array}$ & $\begin{array}{l}\text { Plate-scan } \\
\text { cycles/mm }\end{array}$ \\
\hline \multirow{2}{*}{ MTF of 0.5} & $\begin{array}{l}1.27 \text { slit } \\
\text { 1.22 edge }\end{array}$ & 1.30 slit & & 1.92 slit & 2.17 slit \\
MTF of 0.1 & 3.42 slit & 3.83 slit & & 4.46 slit & 5.50 slit \\
& 3.27 edge & 3.78 edge & & 4.35 edge & 5.32 edge \\
\hline \hline
\end{tabular}


systems. The slit method has been refined and established over many years as the conventional method to measure the MTF of a radiographic system with high accuracy. ${ }^{6-10,33}$ The edge method has also been recognized as a method to measure the $\mathrm{MTF}^{20,47}$ and applied to computed tomography $(\mathrm{CT})^{28,48,49}$ as well as optical and photographic systems. ${ }^{19,23-27,50-52}$ However, the application of the method to radiographic systems has not been fully developed and its value has been acknowledged in only a limited number of recent publications. ${ }^{17,21} \mathrm{We}$ have shown in this paper that the edge method is an accurate and practical alternative for measuring the MTF of digital radiographic systems.

The physical attributes of an edge test device offer advantages when compared with a slit device. A slit test device is usually made of two polished edges spaced parallel to each other. The slit width is in the order of tens of micrometers (usually $10-12 \mu \mathrm{m}$ ) and its thickness along the direction of the incident $\mathrm{x}$-ray beam is in the order of millimeters (i.e., 2 $\mathrm{mm}) .{ }^{3,6,10,13,14,16,18,33}$ An edge device, in contrast, is made of only one polished edge, and its thickness can be less than a millimeter (i.e., $250 \mu \mathrm{m}$ in this study). Physical imperfections in fabrication of either of these test devices can degrade the precision of the measurement. However, the edge method is theoretically less sensitive to such imperfections. As a hypothetical example, a $1 \mu \mathrm{m}$ imperfection along a typical 10$\mu \mathrm{m}$-wide slit causes a $10 \%$ variation in the slit width. Such variations in the form of non-uniformities along the length of a slit have been encountered experimentally and normalization schemes are incorporated to correct for their degrading effects. ${ }^{14,29}$ By comparison, a similar $1 \mu \mathrm{m}$-level imperfection in an edge device causes only a slight blur to that extend in the measured response which is in most cases inconsequential.

Another physical attribute of the slit and edge devices is their thickness which imposes a requirement on the proper alignment of the test device in the x-ray beam. Slit devices are made of thick attenuator material in order to reduce the transmission through the sides of the slit and allow measurement of the signal in the tails of the LSF. In contrast, an edge device can be made of a much thinner material since the information in the tails of the LSF is amplified by the differentiation step. As a consequence, alignment of an edge test device is less sensitive than a slit device. As an example, a $0.1^{\circ}$ tilt of a typical $10-\mu \mathrm{m}$-wide, 2 -mm-thick slit device with respect to the incident $\mathrm{x}$-ray beam leads to a penumbra of $7.0 \mu \mathrm{m}$ (70\% of the slit width) for both edges of the slit which significantly effects transmission through the slit. By comparison, a $0.1^{\circ}$ tilt of a $250-\mu \mathrm{m}$-thick edge device causes a penumbra of only $0.44 \mu \mathrm{m}$ which is inconsequential.

The measurement of the low-frequency response of a system constitutes another advantage of the edge method compared to the slit method. In the slit method, the data for the low-frequency response is derived from information in the tails of the measured LSF. However, the number of detected quanta in the LSF tails is limited because of low transmission at the opaque regions of the slit device. In order to overcome this difficulty, a combination of two techniques is used, namely multiple-slit exposures, and approximation of the tail region by truncating the LSF and extrapolating the truncated LSF according to an a priori exponential form..$^{9,18}$ Although the LSF extrapolation method can be employed successfully for well-behaved systems ${ }^{33}$ the results can be misleading since the estimated MTF at low spatial frequencies is a result of the extrapolation procedure and not the true behavior of the system. A recent study, for example, reports up to $2.5 \%$ discrepancy in the measured MTF for DSP systems as a result of the extrapolation procedure. ${ }^{14}$ In contrast, a large number of quanta contributes to the tails of the LSF in the edge method. Consequently, the edge method can provide a more accurate estimation of the MTF at low frequencies. We consider the edge method to be the preferred approach for measuring the low-frequency response of a radiographic system.

In contrast, the slit method is superior to the edge method in measuring the high-frequency response of a radiographic system. ${ }^{22}$ This limitation of the edge method is understood to be a consequence of noise amplification associated with the numerical differentiation procedure which is an integral part of the method. ${ }^{25,33}$ In our edge method, a number of processing steps were devised to reduce noise including signal averaging, subpixel binning, local smoothing of the ESF data, and windowing the LSF. The results demonstrate that this deficit of the edge method can be controlled with acceptable noise for spatial frequencies up to 7 cycles $/ \mathrm{mm}$. However, a more rigorous assessment of the performance of a radiographic system might be achieved by utilizing both the edge and slit methods to fully characterize the resolution properties of the system.

It should be noted that the edge method we have used does not measure the very low-frequency response of a system which is often associated with scattered radiation or light glare effects. ${ }^{53}$ In order to control noise in the MTF, we limited the length of the reprojection to $\pm 10 \mathrm{~mm}$ from the edge and employed a Hanning window function in this range. This limits the frequency increments of the MTF to 0.05 cycles $/ \mathrm{mm}$ and the spectral resolution to about 0.1 cycles $/ \mathrm{mm}$. Additionally, this insures that the data analyzed is close enough to the edge to consider the edge infinite in length. The resultant MTF was further normalized to its value at zero frequency. Therefore, in our method, the effects of glare which extend over long distances were eliminated. Other tests ${ }^{33,54}$ can be implemented in conjunction with our method to evaluate the glare response of a system.

It can be concluded that the edge method is a simple and accurate method for measuring the low- and mid-frequency response of the system and in the high-frequency region provides adequate results. The lower susceptibility of the edge method to physical imperfections of the test device and imprecision in the alignment procedure suggests that the edge method is a more practical approach in measuring the MTF of radiographic systems in the field. Even in a laboratory setting, the method might be more applicable when extremely small pixel sizes are used, such as in digital mammography systems. ${ }^{17}$ Consequently, we believe the edge method to be an effective approach for determining the resolution properties of a digital radiographic system. 


\section{CONCLUSION}

A method is described to determine the presampled MTF of digital radiographic systems using a sharp, attenuating edge device. The method provides a reproducible measurement of the presampled MTF. The results are equivalent to those obtained by established slit measurements. The simplicity and practicality of the method allows routine and accurate determination of presampled MTF for digital radiographic systems.

\section{ACKNOWLEDGMENTS}

The authors would like to acknowledge the intimate collaboration of Robert Kulpinski, David Trauernicht, and Bruce Whiting of Eastman Kodak Company in facilitating the experimental comparison study and executing the slit measurements. Additional thanks are due to Sean Hames of Henry Ford Health System, Richard Van Metter of Eastman Kodak Company, Mitchell Goodsitt and Heang-Ping Chan of The University of Michigan Medical Center, and Kimberlee Kearfott of the Department of Nuclear Engineering and Radiological Sciences, The University of Michigan, for helpful discussions. The thoughtful critiques of the referees were greatly appreciated. This work was supported in part by a grant from Eastman Kodak Company.

a) This work was presented in part at the 38th annual meeting of the American Association of Physicists in Medicine, Philadelphia, PA, July 1996.

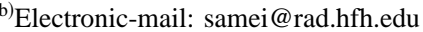

${ }^{1}$ R. H. Morgan, L. M. Bates, U. V. Gopala Rao, and A. Marinaro, "The frequency response characteristics of x-ray films and screens," Am. J. Roentgenol. 92, 426-440 (1964).

${ }^{2} \mathrm{~K}$. Rossmann, "Point spread-function, line spread-function, and modulation transfer function, tools for the study of imaging systems," Radiology 93, 257-272 (1969)

${ }^{3}$ K. Doi, P. C. Bunch, G. Holje, M. Pfeiler, and R. F. Wagner, Modulation Transfer Function of Screen-Film Systems (Bethesda, MD, 1986).

${ }^{4}$ R. A. Sones and G. T. Barnes, "A method to measure the MTF of digital x-ray systems," Med. Phys. 11, 166-171 (1984).

${ }^{5}$ W. Hillen, U. Schiebel, and T. Zaengel, "Imaging performance of a digital storage phosphor system," Med. Phys. 14, 744-751 (1987).

${ }^{6}$ J. Morishita, K. Doi, R. Bollen, P. C. Bunch, D. Hoeschen, G. Sirand-rey, and Y. Sukenobu, "Comparison of two methods for accurate measurement of modulation transfer functions of screen-film systems," Med. Phys. 22, 193-200 (1995).

${ }^{7}$ K. Doi, J. Holje, L.-N. Loo, H.-P. Chan, J. M. Sandrick, R. J. Jennings, and R. F. Wagner, MTF's and Wiener Spectra of Radiographic ScreenFilm Systems, Vol. 1 (U.S. HHS, FDA, Rockville, MD, 1982), No. 828187

${ }^{8}$ M. L. Giger and K. Doi, "Investigation of basic imaging properties in digital radiography. 1. modulation transfer function," Med. Phys. 11, 287-295 (1984)

${ }^{9}$ K. Doi, Y. Kodera, L.-N. Loo, H.-P. Chan, and Y. Higashida, MTF's and Wiener Spectra of Radiographic Screen-Film Systems, Vol. 2 (U.S. HHS, FDA, Rockville, MD, 1986), No. 82-8187.

${ }^{10}$ H. Fujita, K. Ueda, J. Morishita, T. Fujikawa, A. Ohtsuka, and T. Sai, "Basic imaging properties of a computed radiographic system with photostimulable phosphors," Med. Phys. 16, 52-59 (1989).

${ }^{11}$ P. Munro, J. A. Rawlinson, and A. Fenster, “Therapy imaging: A signalto-noise analysis of a fluoroscopic imaging system for radiotherapy localization,' Med. Phys. 17, 763-772 (1990).

${ }^{12}$ U. Neitzel, I. Maack, and S. Gunther-Kohfahl, “Image quality of a digital chest radiography system based on a selenium detector," Med. Phys. 21, 509-516 (1994)

${ }^{13}$ R. Van Metter and R. Dickerson, "'Objective performance characteristics of a new asymmetric screen-film system,' Med. Phys. 21, 1483-1490 (1994).

${ }^{14}$ J. T. Dobbins, D. L. Ergun, L. Rutz, D. A. Hinshaw, H. Blume, and D. C. Clark, "DQE $(f)$ of four generations of computed radiography acquisition devices," Med. Phys. 22, 1581-1593 (1995).

${ }^{15}$ H. Arimura, T. Ikari, M. Okamoto, N. Nakamori, H. Kanamori, H. Kubota, M. Matsumoto, and A. Takigawa, "X-ray voltage dependence of Wiener spectra of quantum mottle obtained by determining detective quantum efficiencies of a screen," Opt. Eng. (Bellingham) 35, 926-937 (1996).

${ }^{16}$ J. Yorkston, L. E. Antonuk, N. Seraji, J. H. Siewerdsen, and Y. El-Mohri, "MTF measurements with high resolution $a$-Si:H imaging arrays," SPIE Med. Imaging 2432, 260-269 (1995).

${ }^{17}$ J. M. Boone and J. A. Seibert, "An analytical edge spread function model for computer fitting and subsequent calculation of the LSF and MTF,", Med. Phys. 21, 1541-1545 (1994)

${ }^{18} \mathrm{~K}$. Doi, K. Strubler, and K. Rossmann, "Truncation errors in calculating the MTF of radiographic screen-film systems from the spread function," Phys. Med. Biol. 17, 241-250 (1972).

${ }^{19}$ R. A. Jones, "An automated technique for deriving MTF's from edge traces," Photograph. Sci. Eng. 11, 102-106 (1967).

${ }^{20}$ J. C. Dainty and R. Shaw, Image Science (Academic, New York, 1974).

${ }^{21}$ I. A. Cunningham and A. Fenster, "A method for modulation transfer function determination from edge profiles with correction for finiteelement differentiation," Med. Phys. 14, 533-537 (1987).

${ }^{22}$ I. A. Cunningham and B. K. Reid, "Signal and noise in modulation transfer function determinations using the slit, wire, and edge techniques," Med. Phys. 19, 1037-1044 (1992).

${ }^{23}$ R. A. Jones and E. C. Yeadon, "Determination of the spread function from noisy edge scans,"' Photograph. Sci. Eng. 13, 200-204 (1969).

${ }^{24}$ A. G. Tescher and H. C. Andrews, "Data compression and enhancement of sampled images," Appl. Opt. 11, 919-925 (1972).

${ }^{25}$ S. E. Reichenbach, S. K. Park, and R. Narayanswamy, "Characterizing digital image acquisition devices," Opt. Eng. (Bellingham) 30, 170-177 (1991).

${ }^{26} \mathrm{P}$. L. Smith, "New technique for estimating the MTF of an imaging system for its edge response," Appl. Opt. 11, 619-622 (1972).

${ }^{27}$ R. M. Simonds, "Two-dimensional modulation transfer functions of image scanning systems," Appl. Opt. 20, 619-622 (1981).

${ }^{28} \mathrm{~S}$. M. Bentzen, "Evaluation of the spatial resolution of a CT scanner by direct analysis of the edge response function," Med. Phys. 10, 579-581 (1983).

${ }^{29}$ J. T. Dobbins, "Effects of undersampling on the proper interpretation of modulation transfer function, noise power spectra, and noise equivalent quanta of digital imaging systems," Med. Phys. 22, 171-181 (1995).

${ }^{30} \mathrm{H}$. Fujita, K. Doi, and M. L. Giger, "Investigation of basic imaging properties in digital radiography. 6. MTFs of II-TV digital systems," Med. Phys. 12, 713-720 (1985).

${ }^{31}$ H. Fujita, J. Morishita, K. Ueda, D. Y. Tsai, A. Ohtsuka, and T. Fujikawa, "Resolution properties of a computed radiographic system," SPIE Med. Imaging 1090, 263-275 (1989).

${ }^{32}$ F.-F. Yin, M. L. Giger, and K. Doi, "Measurement of the presampled modulation transfer function of film digitizers using a curve fitting technique," Med. Phys. 17, 962-966 (1990).

${ }^{33}$ H. Fujita, D.-Y. Tsai, T. Itoh, K. Doi, J. Morishita, K. Ueda, D. Y. Tsai, and A. Ohtsuka, "A simple method for determining the modulation transfer function in digital radiography," IEEE Trans. Med. Imaging 11, 34-39 (1992).

${ }^{34}$ J. M. Boone, T. Yu, and J. A. Seibert, "Sinusoidal modulation analysis for optical system MTF measurements," Med. Phys. 23, 1955-1963 (1996).

${ }^{35}$ C. R. Crawford, "Reprojection using a parallel backprojector," Med. Phys. 13, 480-483 (1986).

${ }^{36}$ C. DiMartini, "Metallography and microstructures," Metals Handbook, Vol. 9 (Americal Society for Metals, Metals Park, NY, 1985), 9th ed. pp. 415-417.

${ }^{37}$ D. M. Tucker and P. S. Rezentes, "The relationship between pixel value and beam quality in photostimulable phosphor imaging,"' Med. Phys. 24, 887-893 (1997).

${ }^{38}$ R. M. Haralick and L. G. Shapiro, Computer and Robot Vision, Vol. 1 (Addison-Wesley, Reading, MA, 1992). pp. 578-588.

${ }^{39}$ J. Princen, J. Illingworth, and J. Kittler, "A formal definition of the hough 
transform: Properties and relationships," J. Math. Imaging Vision 1, 153-168 (1992)

${ }^{40}$ R. O. Duda and P. E. Hart, "Use of the Hough transformation to detect lines and curves in pictures," Commun. ACM 15, 11-15 (1971).

${ }^{41}$ W. K. Pratt, Digital Image Processing (Wiley, New York, 1991).

${ }^{42}$ P. R. Bevington, Data Reduction and Error Analysis for the Physical Sciences (McGraw-Hill, New York, 1969).

${ }^{43}$ R. N. Bracewell, The Fourier Transform and its Applications (McGrawHill, New York, 1986), 2nd ed.

${ }^{44}$ E. Storm, "Calculated bremsstrahlung spectra from thick tungsten targets," Phys. Rev. A 5, 2328-2338 (1971).

${ }^{45} \mathrm{E}$. Storm, "Emission of characteristic $L$ and $K$ radiation from thick tungsten targets,', J. Appl. Phys. 43, 2790-2796 (1972).

${ }^{46}$ T. M. Bogucki, D. P. Trauernicht, and T. E. Kocher, Characteristics of a Storage Phosphor System for Medical Imaging (Eastman Kodak Co., Health Sciences Division, Eastman Kodak Company, Rochester, NY 14650, 1995), Technical and Scientific Monograph No. 6.

${ }^{47}$ G. C. Higgins, "Methods of engineering photographic systems," Appl. Opt. 3, 1-10 (1964).
${ }^{48} \mathrm{P}$. F. Judy, "The line spread function and modulation transfer function of a computed tomographic scanner," Med. Phys. 3, 233-236 (1976).

${ }^{49}$ E. L. Nickoloff and R. Riley, "A simplified approach for modulation transfer function determinations in computed tomography," Med. Phys. 12, 437-442 (1985).

${ }^{50} \mathrm{~B}$. Tatian, "Method of obtaining the transfer function from the edge response function," J. Opt. Soc. Am. 55, 1014-1019 (1965).

${ }^{51} \mathrm{R}$. Barakat, "Determination of the optical transfer function directly from the edge spread function," J. Opt. Soc. Am. 55, 1217-1221 (1965).

${ }^{52}$ N. J. Schneiders and S. C. Bushong, "Single-step calculation of the MTF from the ERF,"' Med. Phys. 5, 31-33 (1978).

${ }^{53} \mathrm{H}$. H. Barrett and W. Swindell, Radiological Imaging-The Theory of Image Formation, Detection, and Processing (Academic, New York, 1981).

${ }^{54}$ J. A. Seibert, O. Nalcioglu, and W. W. Roeck, "Characterization of the veiling glare PSF in x-ray image intensified fluoroscopy," Med. Phys. 11, 172-179 (1984). 